\title{
Unmanned Aerial Vehicles for Environmental Monitoring with Special Reference to Heat Loss
}

\author{
Stanisław Anweiler ${ }^{1, *}$, Dawid Piwowarski ${ }^{1}$, and Roman Ulbrich $^{1}$ \\ ${ }^{1}$ Department of Mechanical Engineering, Opole University of Technology, 5 Mikołajczyka st., 45-758 Opole, Poland
}

\begin{abstract}
This paper presents the design and implementation of device for remote and automatic monitoring of temperature field of large objects. The project aimed to create a quadcopter flying platform equipped with a thermal imaging camera. The object of the research was district heating installations above ground and underground. The results of the work on the implementation of low-cost (below 750 EUR) and efficient heat loss monitoring system. The system consists of a small $(<2 \mathrm{~kg})$ multirotor platform. To perform thermal images micro camera FlirOne with microcomputer Raspberry Pi3 was used. Exploitation of UAVs in temperature field monitoring reveals only a fraction of their capabilities. The fast-growing multirotor platform market continues to deliver new solutions and improvements. Their use in monitoring the environment is limited only by the imagination of the user.
\end{abstract}

\section{Introduction}

Growing share of the municipal sector in energy consumption develops many activities and requirements for energy consumption reduction and the observation of trends necessity. For individual objects there is an energy audit available and the building's energy performance analysis is possible, which significantly contributes to the energy intensity reduction of the economy. In the case of actions relating to larger areas, such as complexes of buildings, districts or even entire cities, such procedure would require a huge financial investment and time. For some time, there are proposals to use the techniques of aerial laser scanning for the urban heat island (UHI) effects [1]. In recent years, attempts are being made to use the unmanned aircraft with thermal imaging techniques. Recent advances in UAVs for civilian use make it possible to regularly monitor environments from the thermophysical point of view, at spatial and temporal scales that would be difficult to achieve using conventional methods. Previous aerial monitoring of thermophysical environments has been expensive and time consuming [2].

This paper presents the design and implementation of device for remote and automatic monitoring of temperature field of large objects. The project aimed to create a quadcopter flying platform equipped with a thermal imaging camera. For this commercially available UAV model was used - 3DR-Iris+ [3]. The object of the research was district heating installations above ground and underground. A series of images to locate the places of heat loss and thus damage to the hull insulation. Additionally a group of public buildings heat loss have been studied.
The aim of the work is the design and implementation of low-cost and efficient heat loss monitoring system. The system consists of a small $(<2 \mathrm{~kg})$ multirotor platform. To perform thermal images micro camera FlirOne with microcomputer Raspberry $\mathrm{Pi} 3$ was used.

In recent years there has been a noticeable increase in the municipal sector's consumption of energy. This is a prerequisite for introducing actions to limit this tendency. One such method is the visualization of heat loss. This is a serious and problematic topic of contemporary engineering when dealing with whole complex of buildings or extensive district heating networks. For individual objects, the subject seems simpler for energy audits and energy performance of buildings.

Taking on the subject of visualization of heat loss in large objects, we face such problems as the difficulty of monitoring due to the surface of the examined objects, the area in which they are located - often industrial objects in hazardous areas with difficult access. Another problem is the cyclical nature of such research.

Periodic thermal imaging of studied objects allows for observation of changes and their tendencies, allows defining defect spots - defective insulation, subassembly or leakage of heating medium. This allows for the development of systematic reviews and periodic repairs.

Another difficulty in monitoring large objects is the way of conducting the study itself. During manual monitoring, by trained staff, it generates high costs and prolongs the time of testing. It also poses a risk to the crew itself because of the area in which the object may be located.

All these aspects create a need for a new method for identifying heat losses in large facilities.

* Corresponding author: s.anweiler@po.opole.pl 


\section{Materials and methods}

In last years laser scanning has been used - LIDAR. This is a modern data mining technique for the numerical model of terrain [4]. The accuracy of the measurement is independent of the lighting conditions. The accuracy range is in the range of $0.1-0.2 \mathrm{~m}$, which is undoubtedly one of the advantages. The disadvantage is the absorption of laser pulses by clouds, fog, water, asphalt and tar.

Research uses a manned aircraft equipped with the necessary equipment. The necessity of having the appropriate permits and licenses to fly in the study areas is one of the first difficulties. Another is the cost of the study itself. The need for a temporary reservation of the airspace in which the flights are to take place is not a matter of delaying the study. If the weather is not proper for fly or the measuring device fails, the flight must be cancelled. It generates losses and high maintenance costs for systematic research.

When analysing the difficulty of visualization of heat loss in large objects, it is concluded that the best method is to take photos from the air. However, the use of manned flying unit in this area may outweigh the costs of research alone. A good unmanned aerial vehicle operated by a terrestrial operator or a pre-defined route can be a good solution - based on GPS coordinates.

The use of unmanned aircraft in environmental monitoring is a relatively new issue. They are used with a positive effect in the monitoring of the animal population, monitoring of areas, in search of missing persons. Even their use in monitoring geothermal areas has been described $[2,5]$. There are many flying models on the market. In addition to price, they distinguish between their capabilities and performance. Flying platform used for study of heat lost should be able to fly on the planned route - e.g. along the heat pipe or overhead line, the possibility of flight in hover mode. This will allow to inspect chimneys co to jest, chimneys, GSM towers and provide a series of images of studied object. The flying model must be able to take off from small and uneven surfaces. Investigated objects may be in the vicinity of other large objects, high vegetation, etc. Its design should allow it to fly in difficult conditions such as high dustiness, high humidity. The great advantage is the possibility of transporting the equipment by one person and carriage in a passenger car, possibly small VAN. It requires the necessity of small size of the platform. In thermal imaging, it is necessary to make them at a constant height to not generate a measurement error. This dictates another criterion when selecting a measuring device.

In recent years, the technology of multirotor models has developed considerably, resulting in stable flight and relatively low maintenance cost [6]. Two families of unmanned aircraft were considered in this research. These were planes, and in it an airplane and a so-called flying wing. The second group were the copters, which included helicopters and multicopters (quadrocopters, hexacopters, etc.). The fundamental difference between them is that in the multicopter there is no precise flight mechanics, only a number of independent electric motors whose rotation is independently changed. Only the rotation speed is responsible for stable flight [3]. Condition of model selection is shown in Table 1. The cost of proposed method is lower than 750 EUR.

Table 1. Condition of model selection

\begin{tabular}{|c|c|c|c|c|c|c|}
\hline $\begin{array}{c}\text { Platform } \\
\text { type }\end{array}$ & $\begin{array}{c}\text { Small/uneven } \\
\text { terrain launch }\end{array}$ & $\begin{array}{c}\text { Dusty/humid } \\
\text { environment } \\
\text { operation }\end{array}$ & $\begin{array}{c}\text { Mobility/one person } \\
\text { transportation }\end{array}$ & $\begin{array}{c}\text { On-board } \\
\text { GPS }\end{array}$ & $\begin{array}{c}\text { Hove } \\
\text { ring }\end{array}$ & $\begin{array}{c}\text { Constant } \\
\text { altitude flight }\end{array}$ \\
\hline Airplane & No & Yes & Yes & Yes & No & Yes \\
\hline Flying wing & No & Yes & Yes & Yes & No & Yes \\
\hline Multicopter & Yes & Yes & Yes & Yes & Yes & Yes \\
\hline Helicopter & Yes & Yes & Yes & Yes & Yes \\
\hline
\end{tabular}

All the criteria are met by the second group of unmanned flying objects - the copter. Due to the presence of precision mechanics on board of the helicopter, multicopters were chosen. Two models were considered. First was the popular DJI Phantom version 3 Standard. However, it has been ruled out for problematic adaptation of non-DJI components. The next model was 3DR Iris +. It has very similar capabilities and performance to the first model. This is an open platform. This means that the user has full access to the source code that dictates the behaviour of the platform on the fly and thus to edit. Iris + allows to connect additional modules such as video accessories, thermal imaging cameras, sensors that may not necessarily have been issued by $3 \mathrm{DR}$.
The Iris + 3DR platform is built on the Pixhawk flight controller, which incorporates sensors such as an accelerometer, a gyroscope, and a barometer. The barometer allows you to fly at a constant height at an accuracy of up to one meter.

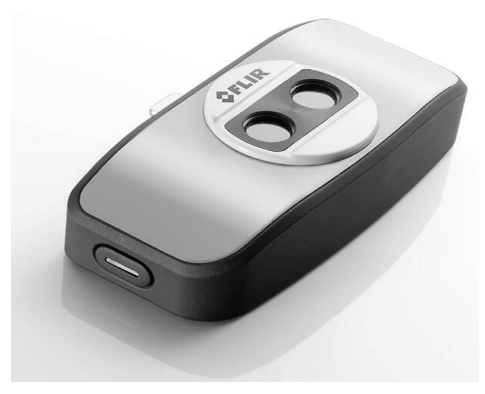

Fig. 1. FlirONE thermal camera 
The thermal imaging camera used, shown in Fig. 1, is a small Flir One device. Its measuring range is -20 to $120^{\circ} \mathrm{C}$. The camera captures two images simultaneously - the first one from the standard camera, the other from the thermal camera, resulting in a $640 \times 480$ image. The measurement resolution is $0.1^{\circ} \mathrm{C}$ but the accuracy is \pm $3^{\circ} \mathrm{C}$. It is dictated by the fact that the camera's optics are able to capture a temperature difference of up to $0.1^{\circ} \mathrm{C}$, but the data processed further by the processor generate error within $\pm 3^{\circ} \mathrm{C}$. The image is refreshed with rate 8.7 $\mathrm{Hz}$.
The camera requires Android or iOS system to work properly. This was resolved by using the popular Raspberry Pi microcomputer. The Android software was installed on the computer and then the camera was attached. At the given stage of the survey, the camera takes pictures at the specified interval of time, e.g. every 5 or 10 seconds. Photos are saved to a memory card in RP3. This provides a collection of photos for further process. A set of described devices located on the test platform is shown in Fig. 2.

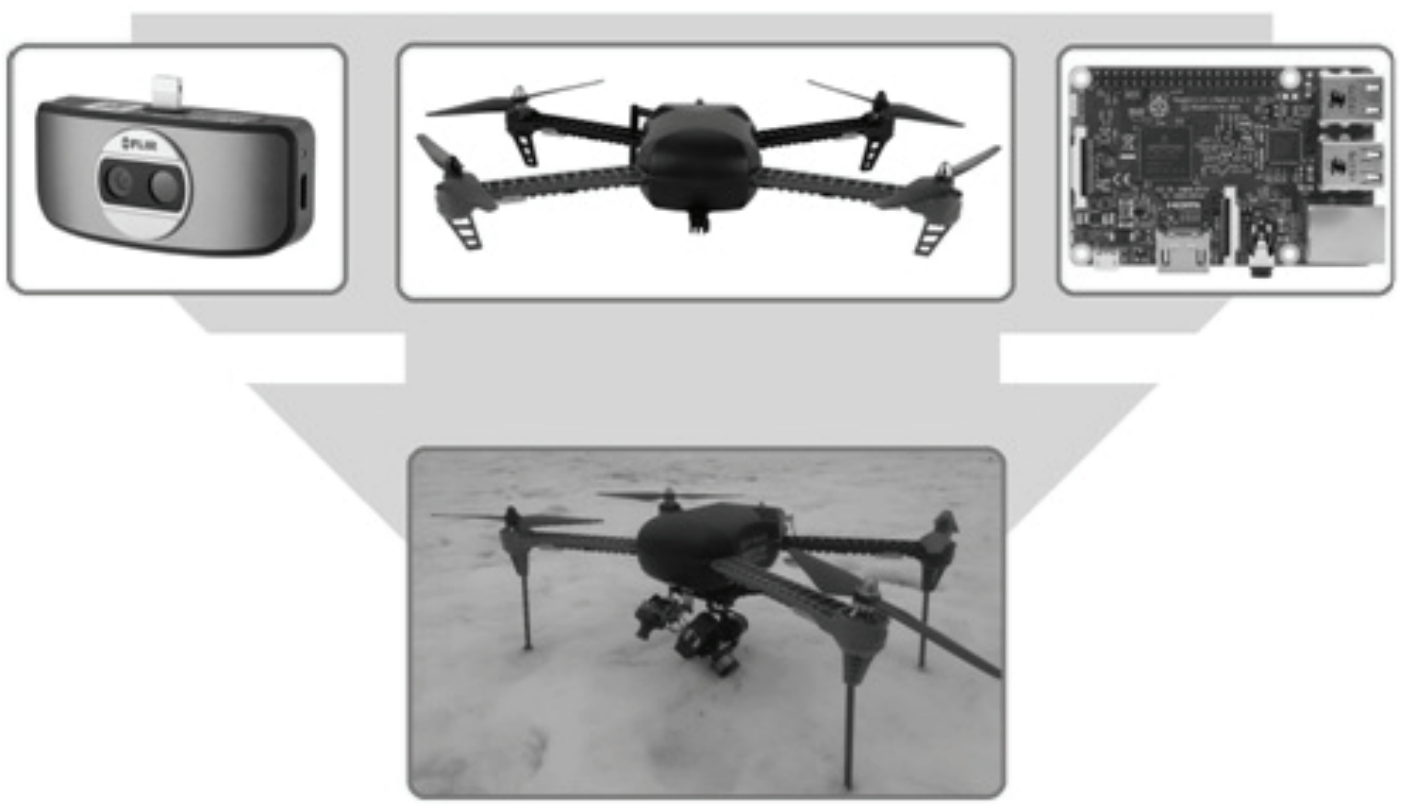

Fig. 2. Quadcopter infrared vision platform assembly.

\section{Results and discussion}

To confirm the usefulness of the camera for the purposes of the research, a series of photographs were made. One of the examined elements was a fragment of ECO Opole heat district. Air temperature during research was $2^{\circ} \mathrm{C}$. Obtained material found that the heat pipe is well insulated, however, we managed to find a few weak points. One was the concrete support through which the heat pipe passed - Fig. 3 .

The thermal images captured the difference in temperature at the spot where the heat exchanger passes

a)

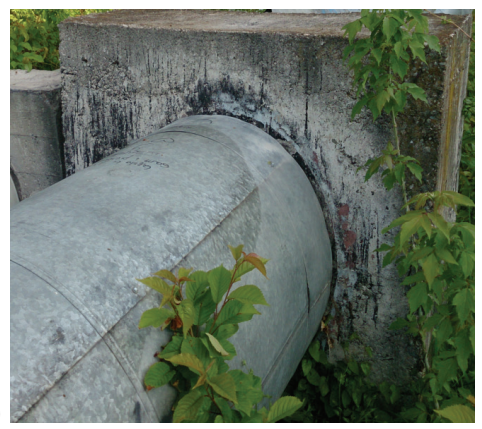

through the concrete block. This generates some loss in transmitted heat, which can only be visualized with the help of a thermal camera.

Next examined object was photovoltaic farm - Fig 4 a) and b).

The photos made it possible to state that the devices are working properly. There is no such thing as hotspots which could suggest that the efficiency of photovoltaic farm is reduced. The presence of hot spots is highly unwanted as it affects the efficiency of the photovoltaic farm [7]. The image that researchers wanted to capture was illustrated by the presence of hot spots in Fig. 4 c).

Fig 3. ECO heating network fragment - concreate support A-general view; B-IR view. 
a)

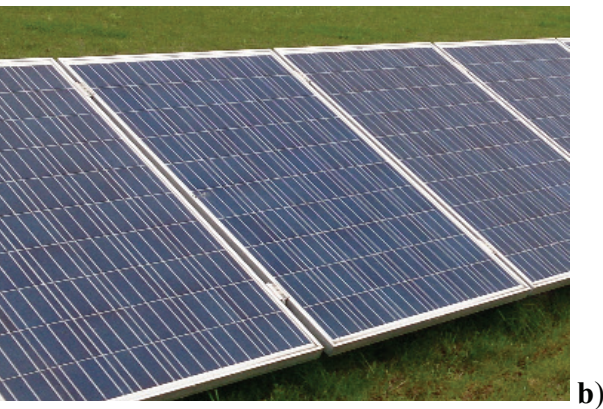

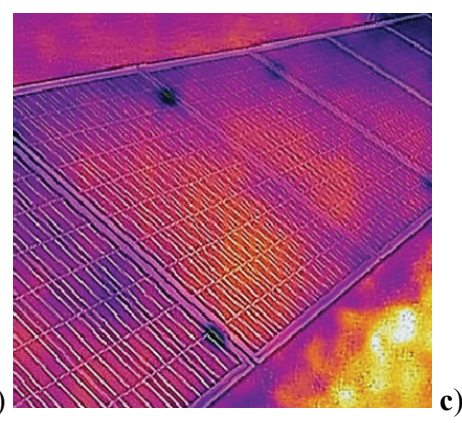

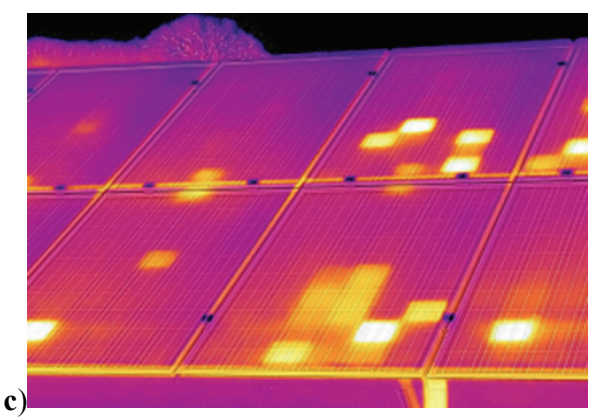

Fig. 4. Examined photovoltaic farm and damaged PV panels. [8]

Photo shows exactly the previously mentioned hot spots, which can only be revealed with thermal camera. As a preliminary study, attempts were made to take pictures of the underground heating pipes. The examined section is part of the ECO Opole heating network. The object consists of two pipes with a diameter of $125 \mathrm{~mm}$ in double insulation. The temperature in the feed pipe is $91^{\circ} \mathrm{C}$ and the return pipeline is about $50^{\circ} \mathrm{C}$. The heating system is shown in Fig. 5

a)

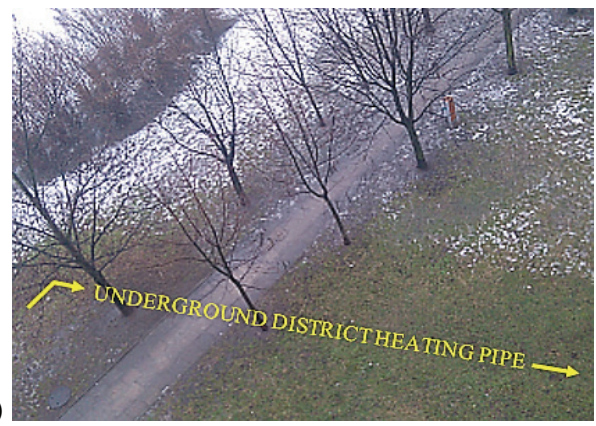

b)

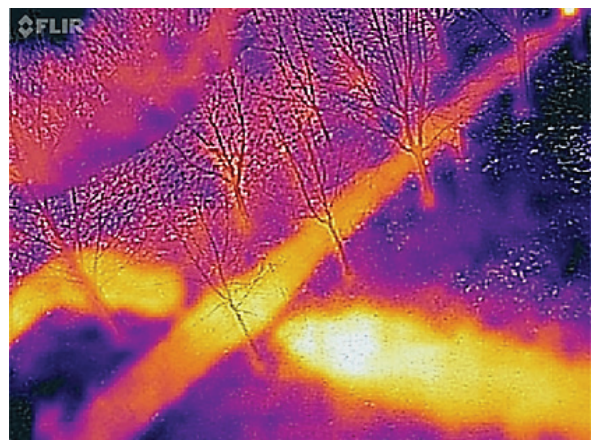

Fig. 5. Underground district heating pipe a-VIS and b-IR footage.

The study was conducted on 05.02.2017r. Under the following atmospheric conditions: temperature $-1{ }^{\circ} \mathrm{C}$, pressure $1020 \mathrm{hPa}$. The weather was windless.

Thanks to taken pictures, are easy to trace the underground heating route and compare it with the GIS map and verify its correctness.

The next stage of the research will be the interpretation of acquired thermal imagery. This will require the introduction of linear temperature field markers, tests for conditions simulated with a heat source of known power and surface temperature, actual conditions and sequences for periodic monitoring of manufacturing plants, building complexes and district heating networks.

\section{Conclusions}

The purpose of the research will be to develop a method of detecting heat losses in large objects - with a great emphasis on industrial objects, heat networks, complexes of buildings. Preliminary results confirm the usefulness of the selected 3DR Iris + platform together with the thermal imaging camera to identify heat losses in the tested objects.

The low cost of the platform maintaining allows periodic measurements. By compiling the collected data, it is possible to indicate defective or wearable elements in advance, planning and systematize periodic inspections and repairs. All of these activities lead to a reduction in the risk of breakdowns, lower maintenance costs for the lines and, consequently, a reduction in the negative impact on the environment.

Using UAVs in temperature field monitoring reveals only a fraction of their capabilities. The fast-growing multirotor platforms market continues to deliver new solutions and improvements. Their use in monitoring the environment is limited only by the imagination of the user.

\section{References}

1. W. Su, Y. Zhang, Y. Yang, G. Ye, Sustainability, 6, 10 (2014)

2. A. Nishar, S. Richards, D. Breen, J. Robertson, B. Breen, Renew. Energ. 86, (2016)

3. S. Anweiler, D. Piwowarski, J. Clean. Prod. 155, 1 (2017)

4. A. Strzałka, PhD Thesis, Opole University of Technology, (2013)

5. M. C. Harvey, J. V. Rowland, K. M. Luketina, J. Volcan. Geoth. Res. 325 (2016)

6. S. Yahyanejad, B. Rinner, ISPRS J Photogramm, 104 (2015)

7. J. Carlson, D. Menicucci, P. Vorobieff, A. Mammoli, H. He,. Appl. Therm. Eng. 70, 1 (2014).

8. Aerial thermal infrared in Melbourne Victoria www.uaviation.com.au/thermal [access: 10.07.2017] 